\title{
LOS PUESTOS DE DIRECCIÓN EN EL ÁMBITO DEPORTIVO: REVISIÓN DE LAS TEORÍAS Y ESTUDIOS SOBRE EL CASO DE LA MUJER
}

\author{
JANA GALLARDo PÉREZ, \\ RoSA MARÍA GONZÁLEZ TIRADOS, \\ ÁNGEL LUIS CLEMENTE REMÓN, \\ JOSÉ ANTONIO SANTACRUZ LOZANO \\ Y MARÍA ESPADA MATEO \\ Madrid
}

Recibido: $17 / 01 / 2013$

Aceptado: 27/03/2013

\section{Resumen}

Desde las últimas décadas, la trayectoria profesional de la mujer y su acceso a cargos directivos ha ganado relevancia como fenómeno de estudio. El presente artículo, aportará el descubrimiento de los diferentes estudios sobre las distintas características y actitudes que dificultan, limitan o impiden el acceso de las mujeres para lograr el potencial laboral máximo que quieran conseguir en las organizaciones y entidades del mercado laboral, y concretamente dentro del sector de la actividad física y el deporte. La metodología seguida en la investigación es cuantitativa y transversal, con una muestra total obtenida de $(\mathrm{N}=401)$. A modo de conclusión de la literatura analizada, se podría resaltar que las oportunidades de trabajo, el acceso o la promoción de las mujeres dentro de una organización o entidad se ven influenciados por las características personales, es decir, de la conciliación del trabajo, la familia y el hogar.

Palabras clave: alta dirección, género, acceso, mercado trabajo, liderazgo.

\begin{abstract}
Since decades, the professional career of women and their access to decision-making positions has gained relevance as a phenomenon of study. This article will provide the
\end{abstract}


discovery of different studies on the various features and attitudes that impede, limit or prevent the access of women to achieve the maximum employment potential that want to get in the organizations and entities of the labour market, and specifically in the field of physical activity and sport. The methodology used in this study is trasversal and quantitative with a final sample of $(\mathrm{N}=401)$. By way of conclusion of the analyzed literature, it could highlight that job opportunities, access or the promotion of women within an organization or entity are influenced by personal characteristics, i.e., the reconciliation of work, family and home.

Keywords: high direction, gender, access, labour market, leadership. 


\section{Introducción}

La progresiva incorporación de la mujer al mercado de trabajo, implica uno de los procesos más importantes de cambio producidos durante el siglo pasado ${ }^{1}$, simplemente fijándonos en los informes del Instituto Nacional de Estadística $^{2}$, se puede ver esta mejora. En cambio, siguen exisistiendo muchas distancias que separan a hombres y mujeres, es decir, se aumenta el número de mujeres trabajando, pero con matices, ya que el $73 \%$ de ellas son separadas y divorciadas, lo que implica mayor cargar familiar, el 52,3\% de los varones declaran que es su pareja quien se encarga del cuidado de los hijos menores de 3 años durante su jornada laboral, mientras que esto ocurre en un 18,5\% de las mujeres. En los casos en los que pueden compatibilizarse las jornadas laborales de ambos cónyuges, con el cuidado de los hijos, el 32,4\% de las mujeres ocupadas y cuyo cónyuge trabaja, declaran que asumen solas el cuidado de los hijos menores de 14 años, fuera del horario laboral, frente al 16,8\% en el caso de los varones que se encuentran en la misma situación. Al mismo tiempo, el $78 \%$ de los varones y el $61,74 \%$ de las mujeres, manifiestan compartir con su cónyuge o pareja la tarea del cuidado de los hijos.

Las principales causas para explicar los porqués de el dificil acceso de las mujeres a puestos de responsabilidad, se pueden enmarcar en las siguientes hipótesis ${ }^{3}$ : la primera enuncia que las mujeres no disponen de la experiencia laboral necesaria para alcanzar puestos de dirección. La segunda propone ${ }^{4}$ que existe una falta de interés o factores motivacionales para acceder a puestos directivos por tener una concepción «altruista-afectiva» del trabajo y por último, que es la propia mujer la que rechaza la incorporación a esos trabajos.

1. GONZÁLEZ TIRADOS, Rosa. «El problema de género en las estructuras empresariales españolas del siglo XXI», en Mujeres y Economía. Instituto de Ciencias De La Educación, 852, (2010), pp. 113-125.

2. «www.ine.es», consultado el 18/09/2010.

3. Selva, Clara, SAHAgún, Miguel Angel., y PAllarÈs, Susana. «Estudios sobre trayectoria profesional y acceso de la mujer a cargos directivos: Un análisis bibliométrico». Revista De Psicología Del Trabajo y De Las Organizaciones 27(3) (2011), pp. 227-242.

4. CASHMORE, E. Making sense of sport. New York: Routledge, 2000. 
En el ámbito deportivo la situación es parecida, las mujeres han encontrado el mundo del deporte dominado y controlado por los hombres ${ }^{4}$. Este hecho, se ha reflejado en la mayoría de las instituciones de la sociedad. En el pasado, las primeras leyes disponían el control de la sociedad en general (es decir, la propiedad, las mujeres no podían poseer, operar un negocio, votar... ${ }^{5} \mathrm{La}$ institución del deporte no fue diferente, ya que las mujeres no accedían a los eventos deportivos con frecuencia y, cuando lo hacían, rompiéndose esa barrera, fueron disuadidas de participar. Como consecuencia de esta dominación masculina, las mujeres han sido limitadas e incluso a veces excluidas de la participación ${ }^{6}$. Por otra parte, las mujeres que han sido capaces de romper la barrera de participación, han sufrido una insuficiente representación y sesgada cobertura por parte de los medios deportivos ${ }^{7}$. Teniendo en cuenta algunos datos encontrados en este ámbito, el 20\% de las mujeres tiene licencia federativa frente al $80 \%$ de los hombres ${ }^{8}$, esto trae como consecuencia la menor participación de la mujer en los Juegos Olímpicos, desde sus inicios, cuando no podían participar, hasta hoy en día, donde su participación está aumentada, pero sigue siendo inferior, en total el 86\% de los participantes han sido hombres, frente al $14 \%$ de las mujeres ${ }^{9}$. Estos datos también se reflejan en el mundo laboral deportivo, siguiendo a Martínez del Castillo $^{10}$, las mujeres están presentes en el sector público deportivo en un $22,1 \%$ de los puestos de trabajo, en el sector de voluntariado en un $16 \%$ y en el sector comercial en un $22,5 \%$, según estos porcentajes, se puede señalar que existe una infrarrepresentación. Siguiendo dentro de este contexto, esta baja representación también ocurre en los puestos de trabajo más altos, ya que de los 8.242 puestos de responsabilidad de los órganos de gobierno de las federaciones, 7423 están ocupados por hombres y tan solo, 819 son mujeres, es decir, el 9,94\% del total, no llegando a alcanzar la cifra impuesta por el Comité Olímpico Internacional [COI], del 10\% para el año 2000 «Comisión

5. COAKLEY, Jay. Sport and society: Issues and controversies. Boston: McGraw Hill, 2001.

6. SAGE, G. H. Power and ideology in american sport: A critical perspective. Champaign: Human Kinectics, 1998.

7. KinkEMA, K. M., \& Harris, J. C. Media Sport studies: Key research and emerging issues, en Media Sport. London: Routledge, 1998.

8. García Ferrando, Manuel. Los españoles y el deporte 1980-1995. Madrid: CSD-Tirant lo Blanch, 1997.

9. CONSEJO SUPERIOR DE DEPORTES. El deporte español ante el siglo XXI. resúmenes, cifras y propuestas. Madrid: Ministerio de Educación y Ciencia, 2000.

10. MARTínez DEL CASTILlo, Jesús. La estructura ocupacional del deporte en España. encuesta realizada sobre los sectores de entrenamiento, docencia, animación y dirección. Madrid: Consejo Superior de Deportes, 1991. 
Mujer y Deporte» ${ }^{11}$. Históricamente, siguiendo el artículo de Pedersen y Warren $^{12}$, los hombres tienen casi exclusivamente el control deportivo ${ }^{13}$. Si bien, este control ha sido evidente en el campo de juego «Sage» ${ }^{14}$ y en la cobertura de los medios de comunicación «Kinkema y Harris» ${ }^{15}$, también ha sido frecuente en todos los niveles de la administración de posiciones en el deporte «Coakley» ${ }^{16}$.

Así pues, la realización del presente artículo conlleva plantear una descripción de la literartura existente en materia de puestos de responsabilidad y liderazgo desde la perspectiva de género.

\section{Teorías feministas}

Para contextualizar el objeto de estudio, se ha partido de las teorías feministas y de género, que han dado forma a la comprensión de las relaciones de género, como un componente fundamental de las relaciones sociales más amplias. Las teorías feministas, tiene como objetivo analizar las condiciones que moldean la vida de la mujer y, explorar la comprensión cultural de lo que significa ser una mujer ${ }^{17}$.

Las doctrinas ideológicas y políticas que inspiraron la Revolución Francesa y los cambios económicos y sociales producidos por la Revolución industrial, sirvieron para establecer las bases de los movimientos feministas, que lucharon por los derechos de la mujer y por su emancipación a lo largo de los siglos XIX y $\mathrm{XX}$, desarrollándose más fuertemente y más pronto en los países más industrializados y de tradición prostestante o librepensadora ${ }^{18}$. Así, las teorías feministas pueden dividirse o concentrarse todas en tres corrientes, según Aitchison ${ }^{19}$, la

11. COMISIÓN MUJER Y DEPORTE (COE). «Mujeres en los órganos de gobierno de las organizaciones deportivas españolas». Instalaciones Deportivas XXI, 146 (2007), pp. 72-78.

12. Pedersen, Paul y WarRen, A. «Successful when given the opportunity: Investigating gender representation and success rates of interscholastic athletic directors». Physical Educator, 62(4), (2005).

13. CASHMORE, Ellis. Op.cit, p.4.

14. SAGE, G. H. Op.cit., p.4.

15. KinKemA, K. M., y HARRIS, J. C. «MediaSport studies: Key research and emerging issues» en MediaSport, London: Routledge, (1998), pp.27-54.

16. COAKLEY, J. Op.cit, p.4.

17. JACKSON, Stevie., y JONES, Jackie. Contemporary feminist theories. New York: New York University Press, 1998.

18. García Ferrando, Manuel., y Lagardera, Francisco. Sociología del deporte. ciencias sociales. Alianza, 1998.

19. AitCHISON, Cara. «Feminist and gender research in sport and leisure management: Understanding the social-cultural nexus of gender-power relations». Journal of Sport Management, 19 (2005), pp. 422-441. 
primera corriente explicaría el sufragismo, movimiento en torno a los siglos XIX y XX, la segunda corriente contendría el Feminismo Liberal, Radical y las perspectivas sociales feministas en los años 1960 y 1970, y por último, la tercera corriente, estaría influenciada por el Postestructuralismo, Postmodernismo y Postcolonialismo. De este modo, se procederá a explicar brevemente, la filosofía de cada teoría femeninsta.

La teoría femenista basada en la antropología, según Moore ${ }^{20}$, explica que las diferencias particulares en la biología reproductiva de los hombres y las mujeres, rara vez funcionan como base de las diferencias sociales entre ellos, o en la comprensión cultural de la diferencia de género. Los autores, McCormack y Strathern, ${ }^{21}$, argumentan que la crítica antropológica no se equipara al dominio de la naturaleza, algo que se ha suscrito a los paradigmas de la sociedad, incrustándose en su cultura como algo biológico, dejando a las mujeres excluyentes de la significación o de la práctica. Por lo tanto, la preocupación que surge de este hecho, es que si no somos capaces de entender otras posibilidades culturales, nos limitaremos a reproducir las imágenes (incluso los estereotipos) de nosotros mismos cuando nos fijemos en los demás. Además, es interesante observar que en gran medida, estos modelos también se acercaron a los límites de género como un concepto heurístico.

La teoría del feminismo social o Estructuralismo, surge en la década de los $80^{22}$. Siguiendo con la Teoría Social, la subordinación de las mujeres podría ser vista como de origen social, ya que no es dada por la naturaleza, ni es un rasgo accidental de las relaciones entre hombres y mujeres. La teoría del feminismo Social fue creada para entender las distancias entre los hombres y las mujeres, y analizar el poder del hombre sobre la mujer. Esta premisa básica recoge que la dominancia por parte del hombre se deriva de la estructura social, política y económica específica de cada sociedad ${ }^{23224}$.

El Feminismo Radical, ve también estas perspectivas, pero considera que tanto el patriarcado y el capitalismo ofrecen un sistema doble de opresión hacia la mujer. Prestando especial atención a las construcciones patriarcales de la sociedad a través de estereotipos sexuales, el heterosexismo y la heterosexualidad obligatoria, la institución del matrimonio, y las prácticas de la

20. MOORE, H. A passion for difference. Oxford: Polity, 1994.

21. MACCORMACK, C., y STRATHERn, M. Nature, culture and gender. Cambridge.: Cambridge University Press, 1980.

22. AitCHison, Cara. Gender and leisure.social and cultural perspectives. London: Routledge, 2003.

23. JACKSON, Stevie. Op. Cit, p. 6.

24. Aitchision, Cara. Op. Cit, p.6. 
pornografía, la prostitución, la violación, el abuso sexual de mujeres y niños, y otras formas de abuso de poder incluida la violencia doméstica. Sin embargo, una crítica radical del patriarcado, es que tiende a considerar las relaciones de poder como un juego que no puntúa, «zero-sum game» en el que el poder se consigue a expensas del otro ${ }^{25}$, en este caso, a expensas de las mujeres.

El feminismo Liberal tiene por objetivo, mejorar las oportunidades de las mujeres dentro de las estructuras y sistemas ya establecidos, pero no puede proporcionar una perspectiva feminista adecuada o completa desde la que abordar la cuestión de la construcción del conocimiento, la legitimación y reproducción, puesto que da un reconocimiento insuficiente de las estructuras y de las culturas que sustentan el conocimiento y el poder de género, ya que la posición feminista liberal, localiza la situación subordinada de las mujeres en la sociedad, dentro de las estructuras públicas, como el sistema educativo y el sistema jurídico, y no acepta la opresión estructural más profunda y cultural, tanto en el ámbito público como en el privado.

El Postmodernismo es concerniente a la crítica del estudio sobre modernidad, mientras que, el Postestructuralismo, se centra en la crítica a las relaciones de poder resultantes de las estructuras y de la estructuración de la modernidad. Por esta diferencia el Postmodernismo se centra en la esfera de las humanidades, mientras que el Postestructuralismo, está dentro de las ciencias sociales, estableciendo un compromiso con las teorías sociales, económicas y de poder cultural ${ }^{26}$. Según Aitchison, la teoría Postestructural pone en tela de juicio la iluminación posterior a las grandes narraciones, que han servido para construir el mundo en categorías dualistas de los sectores público y privado, entre hombres y mujeres, trabajo y ocio, la cultura y la naturaleza, hacia uno mismo y hacia el otro. Por lo tanto, el Postmodernismo niega la posibilidad de una ciencia objetiva, que descubra las leyes de una realidad que existe independientemente, y que repudia la búsqueda de principios éticos, universales y racionales, que rechaza la existencia de una categoría aparte de la estética, que esté separada de los reinos de la ciencia, la ética o de la práctica cultural ${ }^{27}$, es decir, de una realidad explicada por hombres y para hombres.

La teoría feminista Post-colonial ha sido muy influyente: en primer lugar, se ha producido una "globalización» de la teoría feminista general; la teoría feminista ha pasado de ser una preocupación pequeña, con las mujeres blancas, de clase media, de habla inglesa, a una atención hacia las mujeres en

25. Aitchision, Cara. Op. Cit, p.7

26. Aitchision, Cara. Op. Cit, p.6.

27. Waugh, Patricia. «Postmodernism and feminism», en Contemporary feminist theories. New York: New York University Press, 1998. 
diferentes contextos nacionales y culturales; hecho que ha provocado una crisis productiva en el principal pensamiento feminista negro, lo que ha conducido a las feministas occidentales, en particular, a pensar que están hablando de «mujer» a «mujer», y no al hecho de hablar con otra persona ${ }^{28}$. En segundo lugar, también se ha abordado la cuestión de género, ya que en la teoría dominante Post-colonial, la cuestión de género, estaba bastante descuidada. En tercer lugar, y quizás el punto más importante, la teoría feminista Postcolonial ha comenzado a establecerse como una forma de análisis por derecho propio, vista como una crítica del feminismo occidental ${ }^{29}$. Tal perspectiva es vital para contrarrestar la visión del mundo del feminismo blanco, que ha sido acusado por algunas feministas de color, de ser intrínsicamente racista ${ }^{30}$.

\subsection{Teorías explicativas de la segregación ocupacional por sexo en el mercado laboral}

La estructura del mercado laboral, en base a las relaciones en el lugar de trabajo, el control de la naturaleza del proceso y la relación salarial, son subyacentes y están afectados por los símbolos de género, los procesos de género identificados, y las desigualdades materiales entre mujeres y hombres. Estos procesos están relacionados con la compleja estructura de clases. Hay que decir que, cuando en una organización existen políticas de género, significa que las ventajas y las desventajas, la explotación y el control, la acción y la emoción, el significado y la identificación, se modelan a través y en términos, de una distinción entre masculino y femenino ${ }^{31}$. Según Acker ${ }^{32}$, el género actúa como un elemento básico constitutivo en la familia y en el parentesco, pero, de forma menos evidente, ayudando a enmarcar las relaciones subyacentes de otras estructuras, incluidas las organizaciones complejas.

Además, la inequidad de género está presente en la mayoría de los campos profesionales y no aparece de forma aislada, sino que también, forma parte del tejido social. La problemática de este tema, es la predisposición de que es lo «mejor» para hombres, mujeres y para la sociedad, llevando a crear diferentes ideales dentro de las propias personas y dentro del trabajo, generando estas

28. Mills, Sara. «Post-colonial feminist theory», en Contemporary feminist theories, New York: New York University Press, 1998.

29. MiLLS, Sara. Op.cit, p. 8.

30. SimMONDS, F. N. «Difference, power and knowledge: Black women in academia» en Working out: New directions for women's studies. London: Falmer, 1992.

31. Ibíd., p.7.

32. ACKER, Joan. «Hierarchies, jobs, bodies: A theory of gendered organizations». Gender and Society., 4(2),(1990), pp. 139-158. 
inequidades de género ${ }^{33}$. La labor que realizan las mujeres sin estar bien entrenadas o pagadas por ella, tales como las tareas domésticas y la crianza de los hijos, siempre han sido indispensables para la sociedad. La división sexual del trabajo, no es una reliquia obsoleta del pasado, sino el producto y el fundamento de la sociedad actual. Aunque se ha dado a las mujeres el acceso al empleo, no se les ha liberado de su responsabilidad en la familia ${ }^{34}$

Desde 1920 hasta 1970, incrementando el número progresivamente, son muchas las listas donde se dibuja la ausencia de mujeres en posiciones de liderazgo. Esto es una consecuencia de la «regla de oro», que dice que cuanto mayor sea la posición, menos mujeres habrá. Se aplica en todo el mundo, con una desviación en mayor o menor medida a la política, la administración, la cultura y la ciencia así como, del mercado de trabajo ${ }^{35}$.

En Alemania la proporción de mujeres en posiciones de liderazgo dentro de la industria, los negocios y la administración pública está entre el 0,6\% y el $13,5 \%$, y en los puestos más altos, la presencia femenina es de un $5 \%$. En concordancia con la Comisión Europe ${ }^{36}$, el desequilibrio entre hombres y mujeres en posiciones de liderazgo está presente, tanto en el área política como en la económica. Menos de un tercio de los directivos son mujeres, y dentro de los consejos administrativos de las 50 empresas europeas más grandes, sólo hay una mujer por cada 10 hombres. En los Parlamentos Nacionales, la proporción de mujeres, es solo del 24\%, en el Parlamento Europeo la representación es del 33\%. Similares jerarquías de género han sido encontradas en el tercer sector, incluyendo las organizaciones deportivas ${ }^{37}$.

Siguiendo con el estudio de la Organización Internacional del Trabajo de Wirth ${ }^{38}$, determina que en los puestos de trabajo generales relacionados con la gestión, la mujer esta representada en un $25 \%$, pero que sí se analizan los diferentes niveles dentro de la estructura institucional, la presencia femenina en el nivel más alto, es de un $5 \%$.

33. Rapoport, R., BAilyn, L., Fletcher, J. K., y Pruitt, B. H. Beyond work-family balance. advancing gender equity and workplace performance. San Francisco: Josey-Bass, 2002.

34. PFISTER, Gertrud. «Dropping out of leadership. why male and female leaders in german sports federations break off their careers». Sport Management Review, 9(2), (2006), pp. 111-140.

35. PFISTER, Getrud. Op.Cit, p. 10.

36. www. ec.europa.eu/public_opinion/, consultado el 11/11/2010.

37. PFISTER, Gertrud. «Are the women or the organisations to blame? Gender hierarchies in Danish sports organisations». International Journal of Sport Policy, 2(1), (2010), pp. $1-25$.

38. WIRTH, Linda Breaking through the glass ceiling. women in management. Geneva: International Labour Organization, 2001. 
El término «techo de cristal» ha sido acuñado para describir la segregación vertical en las profesiones y las ocupaciones. Dicho término, explica que, todavía hoy, existen barreras invisibles, sumadas a procesos y factores que dificultan el acceso de las mujeres a puestos de alta dirección ${ }^{39}$.

Las teorías explicativas de la segregación ocupacional por sexo en el mercado laboral, se puede subdividir a su vez en tres apartados: 1. La teoría NeoClásica, el modelo del capital humano, 2. Las teorías Institucionales sobre la segmentación del mercado laboral y 3. Las diferentes teorías de Género dentro de este ámbito.

\subsubsection{La teoría Neo-Clásica}

Esta teoría asume que sus trabajadores son eficientes y racionales, por lo tanto, los empleados buscan empleos con mejores sueldos, acordes a su titulación, beneficios dentro de la empresa, intentando maximizar los productos y minimizar el gasto...pero según esto, porqué los trabajadores son pagados como si realizaran un producto marginal. A su vez, esta teoría se divide en dos corrientes,

a) Reflexiones orientadas a la oferta. Este enfoque enfatiza en porqué las mujeres prefieren unas ocupaciones frente a otras, trabajos más flexibles y operados dentro del nivel micro-social. La causa de marginalización de las mujeres dentro del mercado laboral, radica en la capacidad de trabajo, argumentando que las mujeres difieren en gran medida de la capacidad de trabajo, por lo tanto, y siguiendo esta teoría, no es extraño que las mujeres ganen un salario menor, que el de sus compañeros los hombres ${ }^{40}$. La relevancia de ello está fundamentada en diferentes investigaciones, como por ejemplo, el estudio realizado por la Comisión Europea ${ }^{41}$, muestra que el 39\% de las mujeres están trabajando en la Unión Europea, frente aún 61\% que no lo hace. Los hombres trabajan a tiempo completo, en un $75 \%$ frente a un $52 \%$ de las mujeres, sorprende el dato de que las mujeres trabajen en un $30 \%$ a tiempo parcial frente a un $4,6 \%$ de los hombres. Siguiendo con el mismo estudio, dentro de las personas con hijos menores de 15 años que viven en casa, más hombres que mujeres están trabajando. Por el contrario, tres veces más mujeres que hombres que no trabajaban, tenían hijos menores

39. SHINEW, K. J., y ARNOLD, M. L. «Gender equity in the leisure services field». Journal of Leisure Research, 30(2), (1998), pp. 177-194.

40. Anker, Richard. Theories of occupational segregation by sex: An overview. Women, gender and work: What is equality and how do we get there? (2001), pp. 129-156. Geneva: International Labour Office.

41. www.diba.es/libra2003/, consultado el 10/11/2010. 
de 15 años en su hogar. La Comisión Europea, dentro del estudio Eurostat, $2008^{42}$, expone que el 90\% de las mujeres argumenta que los hombres tienen mejores posiciones que las mujeres, porque muchas de ellas tienen que abandonar su formación debido a cuestiones familiares. Además de que un $80 \%$ de las mismas, asegura que la maternidad supone un obstáculo en su carrera profesional. Las razones que aportan las mujeres para no trabajar son, las responsabilidades familiares en un $51 \%$ en Malta, al igual que las mujeres en Alemania y Suiza con un $45 \%{ }^{43}$. Por último, en el artículo de Bialeschki y Henderson $^{44}$, se expone que más del $45 \%$ de las mujeres encuestadas respondieron que la falta de tiempo y el demasiado tiempo que implica la gestión eran algo difícil de conciliar, además de que el 26\% de las encuestadas, indicaba que el sentimiento de culpabilidad y las tareas del hogar eran un gran problema en relación a lo anterior. Aún así, la nivelación entre hombres y mujeres no se produce, debido a la presencia todavía hoy, de conceptos como el «techo de cristal» ${ }^{45}$.

b) Enfoque en función de la demanda: Este enfoque enfatiza en porqué, las personas con responsabilidad de las organizaciones, prefieren contratar a hombres que a mujeres, en determinadas ocupaciones $y$, por otro lado, porque las mujeres y los hombres tienen diferentes oportunidades de promoción y desarrollo profesional dentro de ellas ${ }^{46}$, es decir, mecanismos de mejora de un puesto de trabajo dentros de las organizaciones.

De acuerdo con los investigadores de la década de los 80, la causa mayor de que las mujeres fueran marginalizadas en el mercado laboral era porque se las consideraba trabajadoras poco fiables, debido a sus características como mujer, a su sexualidad, a su capacidad de reproducción, cuidado de los hijos, su menstruación y sus emociones, todo ello hace que la mujer no pueda acceder a puestos superiores, debido a la jerarquía y control masculino.

La hipótesis de la segregación horizontal, según Reskin ${ }^{47}$, es que las mujeres son asignadas a puestos de trabajo que son semejantes al trabajo como ama de casa, así muchas profesiones como por ejemplo, profesoras y secretarias han pasado de ser profesiones masculinas a femeninas, conllevando una

42. www.ec.europa.eu/public_opinion/, consultado el 10/11/2010.

43. COMISIÓN EUROPEA. Op. Cit., p. 12.

44. BiAlesChKI, M. D., y HENDERSON, Karla. «The personal and professional spheres: Complement or conflict for women leisure services professionals». Journal of Park and Recreation Administration, 2(4), (1984), pp. 21-30.

45. Ibíd., p.10.

46. ACKER, Richard. Op.cit. p.12.

47. RESKIN, Bárbara. "Getting it right: Sex and race inequality in work organisations». Annual Review of Sociology, 26, (2000), pp. 707-709. 
pérdida de recursos y prestigio y también, asociándolas a trabajos en función de sus características de mujer ${ }^{48}$. Esto puede ejemplificarse viendo que en una misma profesión u ocupación existen diferentes salarios para hombres que para mujeres, por ejemplo: En el Reino Unido, una encuesta a varias empresas en 1998, mostró una diferencia del 15\% entre las mujeres y los hombres. En el plano directivo, la brecha de género es aún mayor, la diferencia estaba en un $42 \%$. Las encuestas realizadas en otros países durante los últimos años revelan diferencias similares, el 35 por ciento menos para las administradoras en Finlandia, el 50 por ciento menos en Brasil, el 43 por ciento menos en Uruguay, el 12 por ciento menos en Australia y el 16 por ciento menos en los Estados Unidos ${ }^{49}$.

\subsubsection{Teorías institucionales sobre la segmentación del mercado laboral}

Estas teorías comienzan con la aceptación de que las instituciones juegan un papel importante a la hora de contratar, despedir y promocionar, así como, del salario que recibe un trabajador. También se basa, en que el mercado laboral está dividido en varios segmentos, y como esos segmentos ofrecen dificultad de promoción a los trabajadores $«$ Standing ${{ }^{50}}^{5}$ « Anker $»^{51}$. El mercado laboral está dividido en varios segmentos, verticales y horizontales, además de que existe una distinción entre el mercado laboral primario, que es donde se encuentran puestos de trabajo con mayor grado de seguridad, mejor salario y oportunidades de promoción, y el mercado laboral secundario, donde se encuentran las peores condiciones de trabajo. Según «Wirth,2001», las profesiones dominadas por mujeres en los EE.UU. son, peluqueras (90\%), secretarias $(98 \%)$, trabajadoras sociales $(86 \%)$, maestras $(85 \%)$, camareras $(79 \%) \ldots$ mientras que, las profesiones donde la presencia de las mujeres es menor son policías (42\%), abogadas (24\%), informáticas (29\%), pilotos (3\%), arquitectas $(17 \%)$, ingenieras $(8 \%)$, entre otras. Lo que conlleva esta segmentación, es la representación de ocupaciones femeninas y ocupaciones masculinas, destacando el hecho de que las mejores condiciones laborales, es decir, el sector primario, son para los hombres, ya que las empresas prefieren, debido a que tienen que pagar salarios más altos, a personas mayor cualificadas y con más

48. Ibíd., p. 10.

49. WirTH, Linda. «Breaking throught the glass ceiling: Women in management». First International Conference: Pay Equity between Women and Men: Myth Or Reality? Luxemburgo, 2002.

50. Standing, G. «Global feminization through flexible labour». World Development, 27(3), (1989), pp. 583-602.

51. Ibíd., p. 11. 
experiencia «Anker $»^{52} ;$ «Pfister $»^{53}, \mathrm{y}$ «González Tirados ${ }^{54}$. El tejido empresarial propio del género femenino incluiría el sector servicios, el sub-sector comercial y la agricultura, que suelen ser lo social y económicamente menos valorado «Bastida $»^{55}$. Por lo tanto, las profesiones femeninas por excelencia serían las que se consideran de apoyo: enseñanza, enfermería, relaciones públicas, trabajo social y recursos humanos $«$ Clancy $»^{56}$.

\subsubsection{Teorías específicas de género en el mercado laboral}

La básica premisa en la que se basa las teorías de género, es que la mujer se encuentra en una situación desventajosa frente a los hombres, debido al Estado y a la subordinación de la mujer en la familia y en la sociedad. En la mayoría de las civilizaciones, las mujeres son responsables del trabajo de casa y del cuidado de los hijos, mientras que, los hombres aportan el sostén de la economía familiar. Esta división de responsabilidades explica porque las mujeres no consiguen tanta experiencia, educación y formación que los hombres, por lo tanto, quedan apartadas a un segundo puesto.

Los estereotipos son representaciones mentales simplificadas de determinados grupos (en función del sexo, la edad, la etnia, la profesión, el tipo de deporte practicado, etc.), en cuanto a su comportamiento, actitudes, valores, expectativas, etc. que terminan configurando una etiqueta social e indican lo que una persona es, en función de su pertenencia a ese grupo. Se transmiten muy fácilmente a través de las relaciones sociales de manera inconsciente y resultan muy resistentes al cambio, a pesar de que puedan ponerse en marcha medidas de corrección y modificación ${ }^{57}$. Las imágenes estereotipadas y las evaluaciones de las habilidades y comportamientos de ambos sexos, son comunes en el esfera pública, así como, en el lugar de trabajo, influyendo en las percepciones, interpretaciones y juicios de las personas, consiguiendo

52. Ibíd., p. 11.

53. Ibíd., p.10.

54. GonZÁlez Tirados, Rosa. "Women in University Education and in the Professional Environment». En Galindo, M.A. y Ribeiro, D. (Eds.).Women's Entrepreneurship and Economics. New perspectives, Practices and Policies.International Studies in Entrepreneurship. Vol. 1000. (2012), pp. 191-210.

55. BASTIDA, M. «¿Una nueva barrera de cristal?» Capital Humano, 207, (2007), pp. 68-76.

56. ClanCY, Susan. «¿Por qué no hay más mujeres en la cima de la escala corporativa: Debido a estereotipos, a diferencias biológicas o personales?». Academia: Revista Latinoamericana De Administración, 28, (2007), pp. 1-8.

57. Mosquera, María José.; y PUIG, Nuria. «Género y edad en el deporte». En M. García Ferrando, N. Puig, F. Lagardera (Comps.). Sociología del deporte, Madrid: Alianza Editorial, (2002), pp. 114-141. 
que estos estereotipos se resistan al cambio. De hecho, muchas profesiones y sobre todo, muchos puestos que requieren posiciones de liderazgo, se espera la correspondencia con el estereotipo masculino, ya que ante la misma posición los hombres tenían un gran estatus, conocimientos y competencias, frente a las mujeres que se las consideraba como «el segundo sexo» y se las atribuía diferentes características enfocadas hacia la empatía, voluntad y hacia la cooperación $\mathrm{n}^{58,59960}$.

Los estereotipos, estarían dentro de la cultura organizacional, que se puede definir como un sistema común de símbolos y significados, en los que las organizaciones se construyen de forma social y se reproducen simbólicamente a través de la interacción de sus miembros (relaciones formales e informales). Por lo tanto, a través del enfoque de análisis de la cultura, se puede hacer explícita los aspectos invisibles, más allá de la estructura formal de la institución, que explican las dificultades de las mujeres para acceder a puestos de decisión ${ }^{61}$.

\section{Teoría del Síndrome de la Abeja Reina}

Esta teoría sugiere que las mujeres se ven amenazadas por otras mujeres, en última instancia por la atención de los hombres, por lo que evalúan negativamente a otras mujeres y el intento de subvertir su éxito. Todor ${ }^{62}$, encontró apoyo a esta teoría cuando observó que las mujeres trabajando en equipo, evalúan el trabajo de otras mujeres más negativamente cuando los hombres estaban presentes, que cuando los grupos estaban compuestos por mujeres solamente ${ }^{63364}$, explican que las mujeres sienten menos respeto, tienen menos amigos y menos contactos con las posiciones más altas que sus compañeros los hombres, y además, esto se produce en mayor medida cuando se tiene mayor educación y mayor rango ocupacional. El síndrome de la Abeja Reina

58. ACHATZ, Juliane, AllmENDingER, J.utta, y HinZ, Thomas. «Sex segregation in organizations: A comparison of Germany and the US.» Paper Presented at the ASA Meeting at Washington D.C. Washington D.C., 2007.

59. Ibíd., p. 11.

60. Ibíd., p. 10.

61. COMISION EUROPEA. Op. Cit. p. 12.

62. TODOR, M. L. «The effect of sexual composition of a group on discrimination against women and sex-role attitudes». Psychology of Women Quarterly, 5(292), (1980), pp. 310.

63. CoOper. V. W. «Homophily or the queen bee syndrome female evaluation of female leadership». Small, Group Research, 28(4), (1997), pp. 483-499.

64. SOUth, S. J., MARKham, W. T., BOnjeAn, C. M., y CORDER, J. «Sex differences in support for organizational advancemen», Work and Occupations, 14(2), (1987), pp. 261-285. 
sugiere que las mujeres de éxito a menudo se niegan a ayudar a las mujeres inferiores en la jerarquía organizacional. En suma, esta posición teórica sobre los patrones de apoyo intergenéricas en el trabajo, sugiere que los hombres reciben más apoyo que las mujeres, del mismo sexo y compañeros de trabajo del sexo opuesto y supervisores.

Según Reis ${ }^{65}$ y Pérez, ${ }^{66}$, el síndrome de la Abeja Reina también influye en el modo de vida de las «abejas reinas», mujeres que superan todos los obstáculos, llegan a ser estupendas profesionales y madres de familia que han abandonado el ideal de «dorada mediocridad». El perfeccionismo de estas mujeres, las lleva a querer convertirse en una mujer que es capaz de tener éxito en su trabajo como un hombre, mientras que mantiene simultáneamente su feminidad y su éxito como madre y esposa. No es suficiente que intenten ser excelentes en su trabajo, su perfeccionismo las lleva a luchar por tener un buen cuerpo, una buena casa y unos hijos perfectos. Intentan hacerlo todo bien y aún así, se sienten atormentadas por la culpabilidad de no dar suficiente tiempo al cuidado de su familia, de su casa y de su carrera.

\section{Género y liderazgo}

En los inicios de la década de los 70, comienzan las diferentes investigaciones sobre género y liderazgo, demostrándose que las mujeres usan los estilos de liderazgo más democráticos que los hombres. Resaltando éstas investigaciones, que sí existen diferencias en los estilos de liderazgo, pero que en las sociedades contemporáneas los estilos de liderazgo realizados por las mujeres son más eficaces ${ }^{67}$. Un examen de las diferencias entre hombres y mujeres en los estilos de liderazgo demostró que aún contrariamente a las expectativas basadas en los estereotipos, no existen diferencias entre sexos en función de la tarea y la estructura de la organización.

Asimismo, otros autores ${ }^{68}$, apuntan que los rasgos del directivo corresponden con los asociados a los roles masculinos y Wajcman ${ }^{69}$, defiende que las mujeres gerentes dejan sus aspectos femeninos a un lado para desarrollar

65. REIS, Sally. We cant change what we dont recognize: Understanding the special needs of gifted females. Girls, women and giftened. (Trillum Press ed.), New York, 1990.

66. PÉREZ, Luz. «Mujeres superdotadas y sociedad: del «burka» al síndrome de la abeja reina». Faisca, Revista de Altas Capacidades, 9, (2002), pp. 35-55.

67. Hoyt, C. L. «Women and leadership». Leadership. Theory and practice, California: Sage Publications, 2007.

68. Selva, Clara., et al., Op. Cit., p. 4.

69. WajCMAn, Judy. Like a man: Women and men in corporate management. Cambridge: Polity press. Cambridge: Polity Press, 1998. 
los atributos que más habitualmente se asocian a los ejecutivos masculinos. De esta manera, existe un acuerdo generalizado en el que el «liderazgo femenino» es un obstaculizador de progreso ${ }^{70}$.

Siguiendo dentro de esta dinámica, las mujeres son menos efectivas cuando están enmarcadas dentro de roles masculinos, por ejemplo dentro de posiciones militares, pero, sin embargo, son más eficaces que los hombres en lo ámbitos de educación, gobierno y servicio social y mucho más efectivas en posiciones medias de gestión donde las características personales están muy valoradas. Sin embargo, existe una barrera invisible que hace que las mujeres no puedan ascender a posiciones de élite, denominada «techo de cristal», término que apareció en el periódico Wall Street Journal en 1986, siendo una similitud al concepto de «Escalera de cristal» utilizado por los hombres en los ámbitos empresariales ${ }^{71}$.

El concepto de «techo de cristal», es un fenómeno global que reproduce la situación en la que se encuentran las mujeres en función de su representación en niveles bajos y en niveles altos, aunque también puede generalizarse dentro de los contextos raciales y éticos, es decir, dentro de las minorías. Otras investigaciones hacen referencia a los conceptos «glas wall»o «muro de cristal» para referirse a los obstáculos que encuentran las mujeres para ser seleccionadas o para promocionar dentro de una escala horizonta ${ }^{72}$. Para explicar dicha infrarrepresentación, se aportan tres tipos de explicaciones: la primera explicaría las diferencias en función del capital humano, ya que el problema de que exista el concepto techo de cristal es que las mujeres están menos cualificadas que los hombres, apareciendo el concepto de «tubería de agua» denominado en inglés (término original) «pipeline problem». Esta analogía de tubería de agua refleja la situación en la que muchas organizaciones se encuentran con respecto a una continua pérdida de talento femenino. En los países del 'Primer mundo', los hombres y mujeres acceden y son contratados, en el sector de servicios profesionales por igual. Sin embargo, existe una evidencia de que las mujeres se pierden dentro de dicha tubería, a través de la interrupción voluntaria a un ritmo dos o tres veces más rápido que los hombres, una vez que han alcanzado la experiencia o a mitad de carrera. La fuga se ilustra al examinar el nivel de participación femenina en cargos de liderazgo. Aunque es difícil de medir debido a la falta de definiciones uniformes, las estimaciones actuales

70. EAGLY, A.H y KARAU, S. «Role congruity theory of prejudice toward female leaders». Psychological Review, 109, (2002), pp. 573-598.

71. HOYT, C.L. Op.cit., p.18.

72. LinEHAN, Margaret. Senior female international managers: Why so few? Aldershot: Ashgate, 2000. 
del número de mujeres en puestos de alto rango indica un porcentaje muy bajo de esas funciones «Gender Advisory Council» ${ }^{73}$. En segundo lugar, las responsabilidades familiares y de la casa, resolviendo y respondiendo a esto, decidiendo no casarse o no tener hijos, o elegir ser una supermujer o intentando sobresalir en todos los papeles,o bien, aceptando puestos de trabajo a tiempo parcial que lo que conllevan es que los problemas de casa-familiares sigan presentes ${ }^{7475776}$. Y en último término, estarían los prejuicios derivados de la incongruencia del rol femenino, ya que es percibido más negativamente, siendo mas determinante en la toma de decisiones. El pensamiento liderativo masculino puede estar estereotipado, pudiendo afectar a las mujeres líderes provocando respuestas contrarias a las esperadas.

\section{Presencia de mujeres en la estructura organizativa deportiva. Perspectiva internacional}

Las actividades físicas están siempre interrelacionadas con las estructuras, normas e ideales de la sociedad, además de que siempre han constituido un espejo de la perspectiva de género, así como, de su jerarquía. En muchos países y en todas las fases de la historia, la mujer ha jugado un papel específico, aunque también marginal, en los juegos tradicionales, la danza y las actividades físicas ${ }^{77}$.

Los medios de comunicación han contribuido en perpetuar la dominación masculina, ya que por un lado, existe una exclusión de las mujeres en la cobertura retransmitida, por otra parte, los eventos deportivos más retransmitidos, como por ejemplo, el tenis y el patinaje artístico, refuerzan las imágenes estereotipas de las deportistas, minimizando los logros de las mujeres, a través de comentarios deportivos masculino ${ }^{78}$. Además, en los medios de comunicación deportivos se tiende a denominar a las deportistas, «damas»

73. www.pwc.com/women, consultado el 1/07/2012.

74. CuADRADO, Isabel y MORALES, José Francisco. «Algunas claves sobre el techo de cristal en las organizaciones». Revista De Psicología Del Trabajo y De Las Organizaciones, 23, (2007), pp. 183-202.

75. SARrió, Maite, BARBERÁ, Esther, Ramos, Amparo y CANDEla, Carlos. «El techo de cristal en la promoción profesional de las mujeres». Revista de psicología social, 17, (2002), pp. 167-182.

76. HoYT, C.L. Op. Cit., p.18.

77. Hartmann-Tews, Ilse. y Pfister, Getrud. Sport and women: Social issues in international perspective. London: International Society for Comparative Physical Education and Sport \& Routledge, 2003.

78. HALBERT, Christy, y LATIMER, Melissa. «Batting gendered language: An analysis of the language used by sports commentators in a televised coed tennis competition». Sociology of Sport Journal, 11, (1994), pp. 298-308. 
implicando impotencia, elegancia y falta de habilidades atléticas, por el contrario, el término hombre, connota competencia, madurez e integridad, reforzando todo ello los estereotipos ${ }^{79}$. Este lenguaje refleja las diferentes actitudes hacia la mujer en su participación deportiva, pero además, refuerza la visión que esto produce sobre lo que es la «realidad» ${ }^{80}$. A modo de ejemplo, según «Escobar y Robles» ${ }^{81}$, en 2004, en EEUU, la retrasmisión emitida en deportes masculinos frente a femeninos fue del $95 \%$ al $1 \%$ y en los medios impresos, del $20 \%$ a $1 \%$.

De los datos expuestos anteriormente y siguiendo a Pfister ${ }^{82}$, podría deducirse que sí no hay participación femenina en el deporte, no puede existir representación femenina en puestos de decisiones deportivas. Las posiciones de liderazgo son consideradas como los órganos principales de las federaciones deportivas e instituciones, independientemente de los recursos de los individuos, la influencia, las actividades o competencias. Los líderes se ocupan en general, de la planificación y coordinación de tareas y actividades, del desarrollo y despliegue de personal, así como, de la evaluación y el control. Debe tenerse en cuenta que, dependiendo del sistema deportivo vigente, las tareas de gestión en el deporte se puede realizar de manera voluntaria o con una jornada a tiempo completo o parcial.

A continuación se exponen una serie de datos para contextualizar la situación anteriormente explicada. Dentro del sector deportivo y la recreación solo el 22\% de las mujeres ocupan puestos a tiempo completo, frente al 31\% de los hombres ${ }^{83}$. Incluso en el ámbito de los servicios culturales, bibliotecas y museos, considerados tradicionalmente como sectores que se ven favorecidos hacia las trabajadoras, las mujeres y los hombres ocupan aproximadamente el mismo número en contratos a tiempo completo; teniendo las mujeres una probabilidad de trabajar a tiempo parcial de cuatro veces mayor que los hombres. En el sector público, las mujeres ocupan sólo el 4,9\% en los puestos de jefe ejecutivo, los más altos cargos en los gobiernos locales, están representadas en un 6,9\% «Local Government Management Board [LGMB] ${ }^{84}$. En el Reino Unido, dentro del sector de ocio, sólo siete de los 146, es decir, un 5\% de mujeres, ocupan puestos de autoridad «LGMB, 1995». A nivel de gestión

79. RICHASON, Lorber. «Gender stereotyping in the English language», en Feminist frontiers III. New York: McGraw Hill, 1993.

80. Halbert, Christy y Latimer, Melissa. Op.cit., p. 20

81. www.mujerydeporte.org/documentos/, consultado el 4/11/2010.

82. Ibíd, p. 10.

83. http://www.ilo.org, consultado el 12/=7/2012.

84. LOCAL GOVERNMENT MANAGEMENT BOARD. Salaries and numbers information. London: LGMB, 1995. 
de las instalaciones, los hombres siguen dominando en hasta un $80 \%$. Las autoridades están intentando poner en práctica la igualdad de oportunidades. La investigación de Aitchison ${ }^{85}$, encontró que el 86,6\% de las encuestadas decía que en su organización había igualdad de oportunidades. Sin embargo, sólo el 55,7\% de ellas, consideraba que su política era eficaz. Todo lo anterior, deja ver un cambio, dentro de las organizaciones, ya que los puestos se están equiparando, existen políticas de igualdad y sobre todo, se aumenta la conciencia social ante este hecho; quizás, los métodos que se están utilizando no son correctos, según Aitchison ${ }^{86}$, se deberían centrar en cambios culturales y no de organización, así, existe falta de valor y de respeto dentro de la organización, falta de visibilidad y un mínimo de aplicación en la política a emplear. Todo esto, se fundamenta con que un tercio de la población encuestada argumentó que, en su organización existía la presencia de «techo de cristal» en un $15 \%$; un 16,3\%, de los encuestados afirmaron que habían experimentado por sus experiencias personales, discriminación sexual en su organización actual. Shinew y Arnold ${ }^{87}$, dicen que aunque las mujeres están representando la mayoría de los nuevos trabajos en gestión, solo el 11\% del personal ejecutivo en servicios públicos de ocio son mujeres. Estas autoras argumentaban que si bien las mujeres constituyen el 57\% de la fuerza de trabajo, el 97\% de los altos directivos son hombres.

El creciente número de mujeres que participaron en competición olímpica no ha dado lugar a una división equitativa del poder y de la influencia. El COI, fue un «club de caballeros» que desde el principio, negó todos los accesos a las mujeres ${ }^{88}$. En 2001, cuando debería haber un porcentaje del 10\% de mujeres en el COI, de 126 miembros solo 11 eran mujeres, es decir, un 8,7\%. Esto también ocurre en diferentes comisiones existentes dentro del COI; el Comité de Ética, por ejemplo, tiene siete hombres y una mujer en calidad de miembros, la Comisión de Comercialización cuenta con 17 hombres y una mujer, la comisión de la Cultura y Educación Olímpica, veintitrés hombres y cuatro mujeres, y el Comité de Solidaridad Olímpica, son quince hombres y una mujer.

No sólo el Comité Olímpico Internacional, sino también los Comités Olímpicos Nacionales [CONs], así como las federaciones deportivas

85. Ibíd, p. 6.

86. Aitchison, Cara. "Women in leisure services: Managing the social-cultural nexus of gender equity». Managing Leisure, 5, (2000), pp. 181-191.

87. SHINEW, K.L, y ARNOLD, M.L. Op.cit., p. 11.

88. PfiSTER, Getrud. «Líderes femininas en organizaciones esportivas. Tendênciasmundiais». Movimento, 9(2), (2003), pp. 11-35. 
internacionales, cuentan con líderes normalmente masculinos. En el 2000, sólo tres de los ciento noventa y nueve CONs, estuvieron presididos por mujeres, menos del $25 \%$ eran mujeres en puestos ejecutivos como vicepresidentes o secretarias generales y el $42 \%$ eran mujeres en las juntas ${ }^{89}$. El mayor número de mujeres participantes en los consejos de los Comités Olímpicos Nacionales, estuvieron en Canadá y Rumania, con cinco cada uno. En 2002, los CONs han alcanzado el objetivo fijado por el COI, es decir, un $10 \%$ de representación femenina. Sin embargo, los cambios en los números no dicen nada sobre los cambios en materia de influencia y poder. Las mujeres, por ejemplo, todavía están excluidas en la participación en reuniones internacionales, como lo revela Gunilla Lindberg, la única mujer en el Consejo Ejecutivo del COI, en un discurso en el congreso anual de la ACNO en Río de Janeiro en el año 2000, donde la representación femenina estuvo en 8 mujeres, dentro de un total de 400 delegados pertenecientes a 192 Comités Nacionales ${ }^{90}$. También, dentro de las federaciones deportivas en todo el mundo, los hombres dominan los Comités de Deliberación y las mujeres tienen, un papel más secundario. Según «Ferris»" $»^{91}$ en el año 2000, el 35,5\% de las sesenta y una federaciones de deportes olímpicos, tenían mujeres en «puestos clave en el órgano ejecutivo», cinco federaciones habían elegido a una mujer como presidenta y tres como secretaria general.

Se podría resaltar que las oportunidades de trabajo y el liderazgo de las mujeres se ven influidos de forma concreta dentro de su situación familiar, porque el liderazgo requiere una gran disponibilidad de tiempo, energía y concentración, además de flexibilidad. Y siendo la responsable en la mayoría de las familias, su tiempo y energía es escasa y esto es un problema para las mujeres directivas, que aún tienen que cuidar del bienestar de sus hijos o de su pareja. Por otra parte, muchos líderes todavía voluntariamente tienen un punto culminante a conseguir en su carrera profesional. Así, las líderes femeninas tienen que llevar una triple jornada; ¿por qué no es de extrañar que muchas mujeres de carrera opten por no comprometerse con las carreras profesionales dentro de las organizaciones deportivas? Es obvio que los clichés, los ideales y los prejuicios, sobre las mujeres y su falta de eficiencia y carisma, son duraderos y generalizados como los resultados encontrados en muchos estudios que analizan esta cuestión ${ }^{92}$.Como consecuencia de ello, las muje-

89. FERRIS, Elisabeth. «Promoting women sports leaders». Olympic Review, February-March, (2000), pp. 29-32.

90. LiNDBERG, Gunilla. «IOC missing the gender target». Play the Game, 17, (2003).

91. FERRIS, Elisabeth. Op.cit., p.23.

92. PFISTER, Gertrud. Op.cit. p.22. 
res durante años se han ido quedando fuera de esos ámbitos, y actualmente tienen dos opciones, continuar fuera o aceptar la hegemonía existente adoptando sus formas; es decir, perdiendo un poco, o bastante, sus identidades sociales femeninas para determinados puestos empresariales. Las políticas de igualdad de oportunidades han demostrado no ser eficaces en el asunto del acceso de las mujeres a los ámbitos de decisión y poder, ya que, entrar en ellos supone elegir el modelo social masculino o cumplir con los dos modelos, el masculino y el femenino, con la carga física y emocional que ello conlleva, produciendo un desgaste personal ${ }^{93}$.

\section{Conclusiones}

Las teorías feministas aportan una visión general de los inicios de los problemas de las mujeres dentro del mercado de trabajo, coincidiendo muchas en la doble opresión que recibe la mujer por parte del Estado y del cuidado de la familia y el hogar.

Las teorías de la segregación ocupacional dentro del mercado de trabajo, enmarcan que los problemas dentro del trabajo, se deben principalmente a la segregación vertical y horizontal que sufren las mujeres, a la capacidad de trabajo y a las diferentes posiciones que ocupan los hombres y las mujeres dentro de una empresa, a consecuencia, en parte, de los prejucios y estereotipos aún presentes y en menor medida, por lo culpa de las propias mujeres (Síndrome de la abeja reina).

El liderazgo supone un elemento diferenciador a la hora de promocionar o acceder a una empresa, demandando los puestos directivos un liderazgo más autocrático y directivo, similar al que comúnmente se asocia a los hombres.

Los datos demuestran que no existen mujeres en puestos de responsabilidad, aunque se hagan iniciativas como las del COI del 20\% de representación femenina en puestos clave, por lo que, siguen presentes barreras que dificultan el acceso de las mujeres a puestos directivos, es decir, el concepto de «techo de cristal» sigue presente en nuestros días, asociándose, además, conceptos nuevos como «muro de cristal», que dificultan la promoción, a nivel horizontal, de las mujeres.

93. GONZÁLEZ TiRADOS, Rosa. «El problema de género en las estructuras empresariales españolas del siglo XXI», en Mujeres y Economía. Instituto de Ciencias De La Educación, 852, (2010), pp. 113-125.

Feminismo/s 21, junio 2013, pp. 91-115 


\section{Referencias bibliográficas}

ACHATz, Juliane, Allmendinger, Jutta y Hinz, Thomas. «Sex segregation in organizations: A comparison of germany and the US». Paper Presented at the ASA Meeting at Washington D.C. Washington D.C, 2000.

ACKER, Joan. «Hierarchies, jobs, bodies: A theory of gendered organizations». Gender and Society, 4(2), (1990), pp. 139-158

Aitchison, Cara. «Women in leisure services: Managing the social-cultural nexus of gender equity». Managing Leisure, 5 (2000), pp. 181-191

Aitchison, Cara. Gender and leisure.social and cultural perspectives. London: Routledge, 2003.

AITCHISON, Cara. «Feminist and gender research in sport and leisure management: Understanding the social-cultural nexus of gender-power relations». Journal of Sport Management, 19 (2005), pp. 422-441.

ANKER, Richard. «Theories of occupational segregation by sex: An overview». Women, gender and work: What is equality and how do we get there? (pp. 129-156). Geneva: International Labour Office, 2001.

BAstida, M. «¿Una nueva barrera de cristal?» Capital Humano, 207 (2007), pp. 68-76.

BiAleschKi, M. D. y Henderson, Karla. «The personal and professional spheres: Complement or conflict for women leisure services professionals». Journal of Park and Recreation Administration, 2(4), (1984), pp. 21-30.

CASHMORE, Ellis. Making sense of sport. New York: Routledge, 2000.

Chant, Silvia y Pedwell, Carolyn. Women, gender and the informal economy: An assessment of ILO research and suggested ways forward. Retrieved 12/07/2012, 2012, fromhttp://www.ilo.org, 2008.

ClANCY, Susan. «¿Por qué no hay más mujeres en la cima de la escala corporativa: Debido a estereotipos, a diferencias biológicas o a escogencias personales?». Academia: Revista Latinoamericana De Administración, 28 (2007), pp. 1-8.

COAKLEY, Jay. Sport and society: Issues and controversies. Boston: McGraw Hill, 2001.

COMISIÓN MUJER Y DEPORTE (COE). «Mujeres en los órganos de gobierno de las organizaciones deportivas españolas». Instalaciones Deportivas XXI, 146, (2007), pp. 72-78.

CONSEJO SUPERIOR DE DEPORTES. El deporte español ante el siglo XXI. resúmenes, cifras y propuestas. Madrid: Ministerio de Educación y Ciencia, 2000.

CuAdrado, Isabel. y Morales, José Francisco. «Algunas claves sobre el techo de cristal en las organizaciones». Revista De Psicología Del Trabajo y De Las Organizaciones, 23, (2007), pp. 183-202

EAGLY, A.H y KARAU, S. «Role congruity theory of prejudice toward female leaders». Psychological Review, 109, (2002), pp. 573-598. 
Eagly, A. H., WoOD, W., y Diekman, A. B. «Social role theory of sex differences an similarities: A current appraisal». The developmental social psychology of gender. New Jersey: Lawrence Erlbaum Associates, 2002.

ESCOBAR, Kika., y ROBLE, Fe. Mujeres en los órganos de gobierno de las organizaciones deportivas españolas., 4/11/2010, fromwww.mujerydeporte.org/documentos/, 2003.

EUROPEAN COMISSION Participation of women and men in decision-making. Retrieved 10/11/2010, 2010, from www.diba.es/libra2003/, 2003.

EUROPEAN COMISSION. Women and european parliamentary elections. Analytical report. Retrieved 11/11/2010, 2010, from ec.europa.eu/public_opinion/, 2009.

EUROSTAT EUROPEAN COMISION. Estadísticas de empleo. Retrieved 8/07/2012, 2011, from http://epp.eurostat.ec.europa.eu/, 2011.

FERRIS, Elizabeth. «Promoting women sports leaders». Olympic Review, FebruaryMarch, (2000), pp.29-32.

GARCía FERRANDO, Manuel. Los españoles y el deporte 1980-1995. Madrid-Valencia: CSD- Tirant lo Blanch, 1997.

García Ferrando, Manuel., y Lagardera, Francisco. Sociología del deporte. Ciencias sociales. Alianza, 1998.

GONZÁlEZ TiRADOS, Rosa. «El problema de género en las estructuras empresariales españolas del siglo XXI». Mujeres y Economía. Instituto De Ciencias De La Educación, 852, (2010), pp. 113-125.

GonZÁlez TiRAdos, Rosa. «Women in University Education and in the Professional Environment». En Galindo, M.A. y Ribeiro, D. (Eds.).Women's Entrepreneurship and Economics. New perspectives, Practices and Policies.International Studies in Entrepreneurship. Vol. 1000. (pp 191-210). New York: Springer, 2012.

HALBERT, Christy., y LATIMER, Melissa. « «Batting» gendered language: An analysis of the language used by sports commentators in a televised coed tennis competition». Sociology of Sport Journal, 11, (1994), pp. 298-308.

HARTMANn-TEWS, Ilse., y PFISTER, Gertrud. Sport and women: Social issues in international perspecitve. London: International Society for Comparative Physical Education and Sport \& Routledge, 2003.

HosHCHILD, Arlie. The managed heart: The comercialization of human feeling. Berkeley: University of California Press, 1983.

Hoyt, C. L.Women and leadership. Theory and practice (pp. 265-300). California: Sage Publications, 2007.

INSTITUTO NACIONAL DE ESTADÍSTICA. Encuesta sobre calidad de vida en el trabajo. Retrieved 18/09/2010 www.ine.es, 2009.

JACKSON, Stevie. y JONES, Jackie.. Contemporary feminist theories. New York: New York University Press, 1998. 
KANTER, R. M. Women and the structure of organizations: Explorations in theory and behaviour. Another voice. New York: Doubleday, 1975.

KINKEMA, K. M., y HARRIS, J. C. MediaSport studies: Key research and emerging issues. MediaSport (pp. 27-54). London: Routledge, 1998.

LindBERG, Gunilla. IOC missing the gender target. Play the Game, 17, 2003.

LINEHAN, Margaret. Senior female international managers: Why so few?. Aldershot: Ashgate, 2000.

LOCAL GOVERNMENT MANAGEMENT BOARD. Salaries and numbers information. London: LGMB, 1995.

Maccormack, C.,y Strathern, M. Nature, culture and gender. Cambridge: Cambridge University Press, 1980.

Martínez del Castillo, Jesús. La estructura ocupacional del deporte en España. Encuesta realizada sobre los sectores de entrenamiento, docencia, animación y dirección. Madrid: Consejo Superior de Deportes, 1991.

Mills, Sara. «Post-colonial feminist theory». Contemporary feminist theories (pp. 113-131). New York: New York University Press, 1998.

MoOre, H. A passion for difference.Oxford: Polity, 1994.

Mosquera, María José y PUiG, Nuria. «Género y edad en el deporte». En M. García Ferrando, N. Puig, F. Lagardera (Comps.). Sociología del deporte, (114141). Madrid: Alianza Editorial, 2002.

Pateman, Carole. El contrato sexual. Madrid: Anthropos, 1995.

PEDERSEN, Paul y WARREN, A. «Successful when given the opportunity: Investigating gender representation and success rates of interscholastic athletic directors». Physical Educator, 62(4), 2005.

PÉREZ, Luz. «Mujeres superdotadas y sociedad: del «burka» al síndrome de la abeja reina». Faisca, Revista de Altas Capacidades, 9, (2002), pp. 35-55.

PFISTER, Gertrud. «Líderes femininas en organizaciones esportivas. tendencias mundiais». Movimento, 9(2), (2003), pp. 11-35.

PFISTER, Gertrud. «Dropping out of leadership. why male and female leaders in german sports federations break off their careers». Sport Management Review, 9(2),(2006), pp. 111-140.

PFISTER, Gertrud. «Are the women or the organizations to blame? Gender hierarchies in danish sports organizations». International Journal of Sport Policy, 2(1), (2010), pp. 1-25.

PIAGET, Jean. The child's construction of reality. London: Rotuledge\&Kegan Paul, 1958.

Rapoport, R., BAilyn, L., Fletcher, J. K. y Pruitt, B. H.. Beyond work-family balance. Advancing gender equity and workplace performance. San Francisco: Josey-Bass, 2002. 
REIS, Sally. M. «We can't change what we don't recognize: Understanding the special needs of gifted females». Girls, women and giftened (Trillum Press ed.). New York, 1990.

RESKIN, Barbara. F. «Getting it right: Sex and race inequality in work» organizations. Annual Review of Sociology, 26, (2000), pp. 707-709.

RESKIN, Barbara. F. y Roos, Patricia P. A. Job queues, gender queues: Explaining women's inroad into male occupations. Philadelphia: Temple University Press, 1994.

RESKIN, Bárbara. F. y RoOS, Patricia. Status hierarchies and sex segregation. Ingredients for women's employment policy. Albany: SunyPress, 1987.

SAGE, G. H. Power and ideology in american sport: A critical perspective. Champaign: Human Kinectics, 1998.

SARrió, Maite, Barberá, Esther, Ramos, Amparo y CANDEla, Carlos. «El techo de cristal en la promoción profesional de las mujeres». Revista de psicología social, 17, (2002), pp. 167-182.

Selva, Clara, Sahagún, Miguel. Angel., y Pallarès, Susana. «Estudios sobre trayectoria profesional y acceso de la mujer a cargos directivos: Un análisis bibliométrico». Revista De Psicología Del Trabajo y De Las Organizaciones, 27(3), (2011), pp. 227-242.

SHINEW, K. J., y ARNOLD, M. L. «Gender equity in the leisure services field». Journal of Leisure Research, 30(2), (1998), pp. 177-194.

SimMONDS, F. N. «Difference, power and knowledge: Black women in academia». Working out: New directions for women's studies. London: Falmer, 1992.

South, S. J., Markham, W. T., Bonjean, C. M., y Corder, J. «Sex differences in support for organizational advancement». Work and Occupations, 14(2), (1987), pp. 261-285.

StAnding, G. «Global feminization through flexible labour». World Development, 27(3), (1989), pp. 583-602, 1989.

TODOR, M. L. «The effect of sexual composition of a group on discrimination against women and sex-role attitudes». Psychology of Women Quarterly, 5(292), (1980), pp. 310.

WAJCMAN, Judy. Like a man: Women and men in corporate management. Cambridge: Polity Press, 1998.

Waugh, Patricia. Postmodernism and feminism.Contemporary feminist theories. New York: New York University Press, 1998.

WIRTH, Linda. Breaking through the glass ceiling. women in management. Geneva: International Labour Organization, 2001.

WIRTH, Linda. Breaking throught the glass ceiling: Women in management. First International Conference: Pay Equity between Women and Men: Myth Or Reality? Luxemburgo, 2002. 